\title{
The Fiocruz Latest Contribution for Understanding Human Tuberculosis Vaccine Development and its Pathogenesis
}

\section{Paulo RZ Antas*}

Laboratory of Clinical Immunology, Instituto Oswaldo Cruz, Fiocruz, Rio de Janeiro, Brazil

Based on 2012 WHO global report, tuberculosis (TB) is a major cause of illness and death worldwide, with increased burden mainly in developing countries. The Stop TB Partnership goals embrace dropping the global TB burden by half in 2015 and eliminating TB as a public health problem by 2050 . Importantly, vaccination is a key approach in reducing the frequency of TB. Mycobacterium bovis bacillus CalmetteGuérin (BCG), the only licensed TB vaccine currently in use, was first administered orally in 1921, and since then many clinical trials in different parts of the world have evaluated the effectiveness of BCG in preventing TB disease. These trials demonstrate that BCG confers steady protection against extrapulmonary $\mathrm{TB}$ in children. However, BCG affords extremely inconsistent defense against pulmonary TB, which accounts for the major burden of global TB mortality and morbidity throughout the world. Therefore, a more successful vaccine is a major global health priority by WHO. The Moreau BCG strain has been employed for some 70 years here in Brazil and lessons from its use should be taken in account for the development or improvement of new TB vaccines. In this venue, the challenges for the subsequent forthcoming years are obvious. It is improbable that any simple immunological correlate of protection will be recognized. Therefore, it is essential our understanding on how BCG confers protection. The vast majority of the current population has been vaccinated with BCG, with the possible requirement for a booster immunization in adulthood for TB protection. BCG Moreau strain also protects against leprosy, meningitis and extrapulmonary forms of TB. Factors related to differences in strain, dosage and BCG administering protocol have been responsible for the variable efficacy. This vaccine is clearly affected by, as yet unclear, host and/or environmental variables. Yet, little is known about the interaction of BCG with human monocytes. Also in preclinical studies, IFN $\gamma$ seems to be required but not sufficient for protection, and the magnitude of the immune response correlates with the degree of protection. However, any immunological correlate may be vaccine and/or disease-stage specific. One of our central goals is to investigate the potential of BCG Moreau strain to induce in vitro specific cell-death in BCG-stimulated monocytes from healthy adults. There was a concomitant release of IL- $1 \beta$ and TNF- $\alpha$, but not metalloproteinase (MMP)-9. In addition, there was an enhancement of monocytes necrosis, but not apoptosis, following BCG Moreau strain stimulation of umbilical vein cells from naïve, neonate population. This pattern was paralleled by different pro-inflammatory cytokine levels, as well as MMP-9 induction when compared to the adults. Those findings support the hypothesis that BCG induces distinct cell-death patterns during the maturation of the immune system and that this pattern might set the stage for a subsequent antimycobacterial immune response that might have profound effects during vaccination. In another small study, the in vitro Th1-immune response of neonates (IFN $\gamma$ ) was shown to be deficient when cells were placed in contact with recombinant antigens by an ELISPOT assay. In fact, the probable impairment was related to a non-specific immune response, since a potent mitogen assayed in parallel as an internal positive control yielded virtually no response in that group. Also, cells from vaccinated adult individuals in matching identical assays, yielded convincing data. The reasons behind this result are speculative; perhaps due to a higher amount of circulating immature immune cells or to a lack of exposure to mycobacterial antigens.

As pointed out above, the production of IFN $\gamma$ guarantees effective T cell-mediated immunity against Mycobacterium tuberculosis infection. However, simply and conventional techniques have compared only the in vitro immune responses to Mycobacterium antigens in terms of IFN $\gamma$ production in PBMC by ELISA. There was an overall profile of agreement in response to recombinant antigens when whole blood and PBMC were compared. Further, we were encouraged to perform that same study now in a broader assessment of serum biomarker, biosignature profiles. Detecting 17 cytokines by a Luminex array system; there was agreement between PBMC and WBA for IL-2, IL5 , IL-6, IL-7, IL-13, IFN- $\gamma$, TNF- $\alpha$, MCP-1and MIP- $1 \beta$. There was evidence toward higher IL-10 and G-CSF plasma production, and higher IL-1 $\beta$, IL-4, IL-12p70, IL-17 and GM-CSF production for PBMC vs. whole blood. These results suggest that, depending on the method employed, PBMC and/or WBA techniques provide fine conditions for the model proposed and thus whole blood cultures are well-suited lowcost proxy-measures during search for serum biomarkers.

Extrapulmonary TB is presumably a marker of immunodeficiency, because median CD4+ counts were lower in that group when related to pulmonary and latently infected patients. In addition, the median baseline cytokine levels were uniformly lower in extrapulmonary patients than both sets of controls. HIV-seronegative adults with previous extrapulmonary TB had lower CD4+ lymphocytes and baseline cytokine production, suggesting a subtle abnormality in innate immune function. After these findings, we searched for genetic variants leading to TB susceptibility. This pilot study among extrapulmonary TB patients with well-characterized immune defects uncovered genetic variants in IL-1 $\beta$, VDR Fok1, and TLR2 associated with an increased risk of extrapulmonary disease. Latter, we identified 6 novel variants not previously known to be associated with extrapulmonary $\mathrm{TB}$, including two SNPs more common in persons with extrapulmonary than pulmonary TB. This provides some support for the hypothesis that the pathogenesis and genetic predisposition to extrapulmonary TB differs from pulmonary disease.

There is a heterogeneous interchange between M. tuberculosis and

*Corresponding author: Paulo RZ Antas, Laboratory of Clinical Immunology, Rio de Janeiro, Brazil, Tel: +55-21-3865-8152; Fax: +55-21-2290-0479; E-mail: pzuquim@ioc.fiocruz.br

Received June 28, 2013; Accepted June 28, 2013; Published July 01, 2013

Citation: Antas PRZ (2013) The Fiocruz Latest Contribution for Understanding Human Tuberculosis Vaccine Development and its Pathogenesis. J Anc Dis Prev Rem 1: e107. doi: 10.4172/2329-8731.1000e107

Copyright: @ 2013 Antas PRZ. This is an open-access article distributed unde the terms of the Creative Commons Attribution License, which permits unrestricted use, distribution, and reproduction in any medium, provided the original author and source are credited. 
Citation: Antas PRZ (2013) The Fiocruz Latest Contribution for Understanding Human Tuberculosis Vaccine Development and its Pathogenesis. J Anc Dis Prev Rem 1: e107. doi: 10.4172/2329-8731.1000e107

the human host. Similar to the varied pathological representations of active TB disease, latency encompasses an assorted array of biological states. The perception of a TB spectrum implies to identify a given position in the spectrum, so that the risk for reactivation and disease progression can be promptly assessed. Along with those studies pointed out above, we have focused lately on unveiling certain aspects of this fascinating disease, particularly the immune response and an in vitro model of prophylactic vaccination. Therefore, the studies in the field are still inconclusive and many questions remain to be urgently answered, particularly the one related to BCG vaccine platform and its consequences for immunization in the population. The studies using "omic" techniques should be continued at the clinical level as well.
Citation: Antas PRZ (2013) The Fiocruz Latest Contribution for Understanding Human Tuberculosis Vaccine Development and its Pathogenesis. J Anc Dis Prev Rem 1: e107. doi: 10.4172/2329-8731.1000e107
Submit your next manuscript and get advantages of OMICS Group submissions

Unique features:

- User friendly/feasible website-translation of your paper to 50 world's leading languages

- Audio Version of published paper

Digital articles to share and explore

Special features:

250 Open Access Journals

20,000 editorial team

21 days rapid review process

Quality and quick editorial, review and publication processing

Indexing at PubMed (partial), Scopus, DOAJ, EBSCO, Index Copernicus and Google Scholar etc

- Sharing Option: Social Networking Enabled

- Authors, Reviewers and Editors rewarded with online Scientific Credits

- Better discount for your subsequent articles

Submit your manuscript at: http://www.editorialmanager.com/acrgroup/ 